\title{
Bioanalysis
}

\section{Proposal for a harmonized descriptive analyte nomenclature for quantitative large-molecule bioanalysis}

\author{
"Rather than to discriminate between free drug or complexed drug ... we propose \\ to discriminate between and provide definitions for active drug, target-binding \\ competent drug and total drug..."
}

\begin{abstract}
Keywords: active drug $\bullet$ free analyte QC concept $\bullet$ target-binding competent drug - total drug
\end{abstract}

It has been well recognized in the scientific community that bioanalysis of therapeutic proteins is not limited to one 'correct' result but several forms of the analyte might be quantified. The expanding numbers of terms for large-molecule analytes in quantitative bioanalysis for preclinical and clinical studies and the inconsistency in nomenclature have often led to confusion. Here, we propose a descriptive nomenclature for large-molecule analytes based on functionality. This might aid in the clarification of the exact nature of the drug fraction that is being analyzed among bioanalysts and within multidisciplinary drug development teams [1]. For large-molecule bioanalysis, drug quantification assays also referred to as PK assays have always been an essential part of preclinical and clinical drug development. A huge effort is undertaken to understand the minimal efficacious dose and to maximize the dosing intervals to reduce development and therapy costs and enhance patient convenience.

There is an increasing awareness that the applied dose, the concentrations of drug in circulation (PK parameters) including the assessment of antidrug antibodies (ADAs) and soluble target or other drug-binding proteins which may reduce the activity of the drug in vivo are key parameters for the understanding of PD markers, efficacy end points and safety observations in individual patients during clinical studies.

It is common understanding that immune responses against the drug occur for basically all large molecules whether they are of animal, humanized or human origin, which to a certain percentage may neutralize the potency of the drug [2]. It is, therefore, obvious that a correlation of only the absolute total drug concentrations to PD, efficacy and safety can be misleading and may not be sufficient to describe the efficacious and safe dose [3]. Consequently, much effort has been invested in the past years to quantify certain forms of the drug molecule, such as the quantification of the subfraction of 'active (potent) drug' molecules, 'free drug' or, as an alternative approach, to understand the impact of ADAs on the potency of drug by, for example, neutralizing ADA assays or ex vivo potency assays [3-6]. Neutralizing assays are, however, only of qualitative nature describing the potential impact of ADAs on the drug and cannot be used to quantify or mathematically calculate the remaining potent fraction of the drug. It has, therefore, been acknowledged that the quantification of active drug or its PD effect is the more relevant parameter during development than the assessment whether a relative subfraction of the drug may be neutralized by ADAs [7,8]. In analogy, the presence of a soluble form of the target or a natural-binding protein may also influence the quantified drug levels. In addition, the drug may also undergo degradation in vivo (e.g., chemical degradation, like deamidation and oxidation or enzymatic digestion or proteolytic cleavage), resulting in degraded drug, which may lead to an inability to exert its full function [9].

Based on that, it appears reasonable and necessary to introduce a descriptive nomenclature for the molecule which is actually

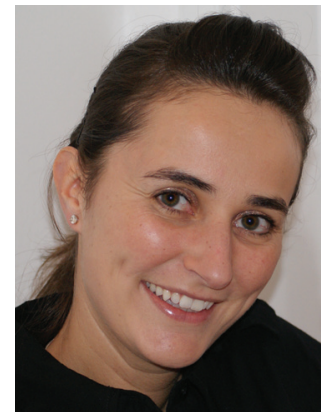

Julia Heinrich

Author for correspondence: Roche Innovation Center Penzberg, Large Molecule Bioanalytical R\&D, Pharmaceutical Sciences, pRED, Penzberg, Germany julia.heinrich@roche.com

\section{Roland F Staack}

Roche Innovation Center Penzberg, Large Molecule Bioanalytical R\&D, Pharmaceutical Sciences, pRED,

Penzberg, Germany

Kay-Gunnar Stubenrauch Roche Innovation Center Penzberg, Large Molecule Bioanalytical R\&D, Pharmaceutical Sciences, pRED,

Penzberg, Germany

\section{Apollon Papadimitriou}

Roche Innovation Center

Penzberg, Large Molecule Bioanalytical R\&D,

Pharmaceutical Sciences, pRED, Penzberg, Germany

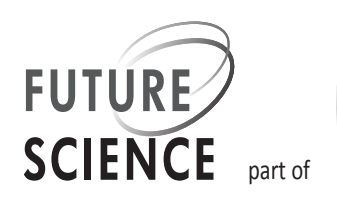


analyzed in a certain assay, in other words, the exact nature of the analyte.

\section{"Quantification of the target-binding competent drug fraction considers the fraction of drug that, in principle, could bind to its target but it does not claim to reflect the real in vivo situation."}

In the literature, multiple terms for different drug species have been used, such as 'total drug,' 'active drug,' 'potent drug' or 'free drug' [5,10], making it difficult to understand what exactly has been analyzed. In addition, we have to be aware that the applied assay or assay technology, including sample preparation, dilution and extraction procedures, as well as the incubation times defines what exact fraction of the drug is being quantified in a specific assay [5,11-12].

Rather than to discriminate between free drug or complexed drug (see Figure 1), with free drug being the drug fraction not bound and complexed drug being the fraction that is bound to a naturally occurring target or binding protein or to ADA in vivo, we propose to discriminate between and provide definitions for active drug, target-binding competent drug and total drug since these are regarded as relevant parameters to describe the drug in a functional context, relevant for establishing correlations to PD or safety. Also, we look into the typical methods applied for bioanalysis of biologics and describe which subfraction of the drug is quantified by the respective assays. For simplicity and clarity reasons, we focus on monoclonal antibodies as the analyte and discuss other more complex biologics (e.g., fusion proteins, bispecific antibodies etc.) only occasionally.

\section{The definition of the term 'total drug'}

In case, functionality of the drug is not assessed by a respective assay and in principle, all subfractions of the drug (i.e., drug isoforms) are quantified, we propose to use the term 'total drug' that comprises all forms of the drug, be it able to bind to its target or not, be it free or complexed and active or degraded. This is, for example, achieved by LC-MS/MS assays that usually quantify a certain signature peptide of the drug after a digestion step. Another way to quantify total drug levels is assays that detect human antibodies in preclinical species by using selective binding to human-specific antibodies [13,14]. It has to be noted that in contrast to LC-MS/MS methods, assays such as ligand-binding assays (LBAs) add information on the integrity of the protein since they confirm that, for example, both heavy chains of an antibody are present if antihuman-Fc antibodies are used for cap- ture and detection. Since binding of ADAs to the regions of capture and detection antibody epitopes or degradation of the drug at these binding sites may occur in some cases such LBA-based assays are not bona fide 'total drug' assays. But still, based on extensive platform knowledge on the stability of the Fc part, it is our opinion to categorize such assays under the total drug category, especially in combination with an acid dissociation step. This step may, for example, be required if a relevant amount of binding proteins (such as the soluble target, binding proteins or ADAs) is present that is able to compete with the assay reagents [11]. In case, only a small (probably irrelevant) fraction of the drug is missed when omitting a dissociation step, the assay can be described as 'quasi-total.' This would, for example, be the case when an ADA assay provides a negative or borderline positive result, so that the probability is low that the ADAs target a significant level of the binding epitope of the capture/detection reagent. Usage of polyclonal antibodies for capture and detection of multiple epitopes would minimize the probability of missing relevant amounts of drug.

\section{The definition of the term 'target-binding competent drug'}

A subfraction of the total drug is the fraction of 'target-binding competent drug' molecules. As the name implies, quantification of these molecules requires the usage of the target as capture or detection reagent, thus assessing the functionality of the drug. Quantification of the target-binding competent drug fraction considers the fraction of drug that in principle could bind to its target but it does not claim to reflect the real in vivo situation. In case of relevant amounts of soluble target or ADA in circulation, the concentration of the target-binding competent drug measured in the assay is lower than the total amount of target-binding competent molecules since an appropriate target capture assay is sensitive to these kinds of drug neutralization and only detects the uncomplexed drug molecules. It may, in contrast, overestimate the amount of active drug in vivo due to dissociation of ADAs from the drug during assay conduct. Quantification of the total amount of target-binding competent drug would require either a dissociation step or long incubation times along with an excess of target reagents to force the equilibrium toward the assay reagents to avoid underestimation of the total target-binding competent fraction. If absence of ADAs or other binding proteins have been shown, it can be argued that the whole fraction of target-binding molecules has been captured in the assay. In this case, the target- 


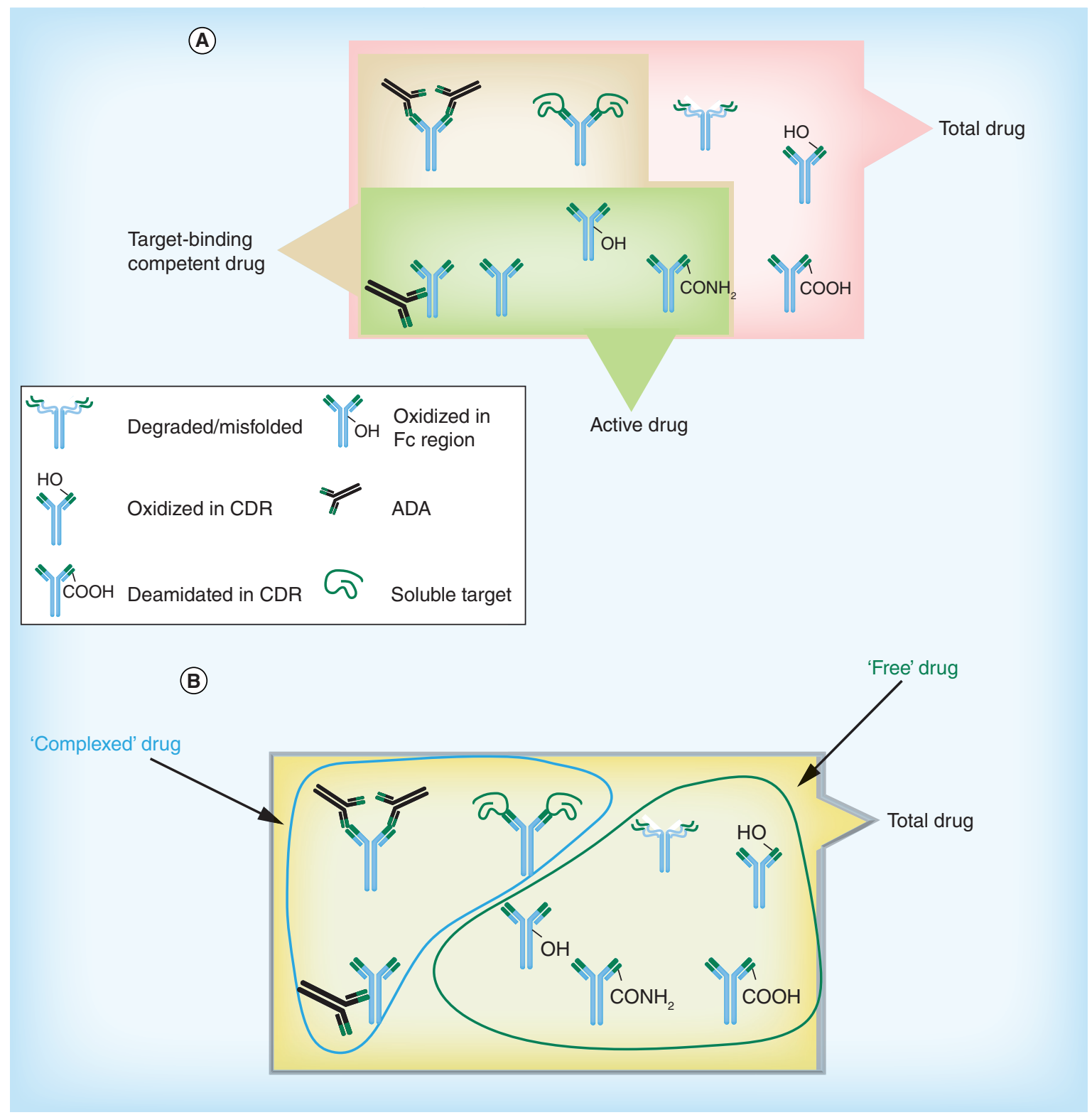

Figure 1. Illustration of different drug fractions to explain the desrciptive analyte nomenclature. (A) Graphic illustration of total drug fraction (pink), the target-binding competent drug fraction (beige) and active drug fraction (green). The total drug fraction comprises all forms of drug, independent of their functionality. The target-binding competent fraction comprises only drug forms that are functional or, in other words, bind to the desired target. Dependent on the assay, not all target-binding competent molecules may be captured. The active drug fraction comprises all functional forms of the drug in vivo, which may require special considerations for the assay conduct to maintain the equilibrium between drug, soluble target and antidrug antibodies. (B) The fraction of complexed drug (blue circle) contains active drug molecules, as well as molecules that are not able to bind its desired target in vivo due to blockage of the binding region. The fraction of free drug (green circle) contains active drug molecules, as well as molecules that are not able to bind its desired target due to degradation, denaturation or other events.

ADA: Antidrug antibody.

binding competent assay quantifies active drug. By contrast, if ADAs or binding proteins are present, a target-binding competent drug assay without additional pretreatment step provides quantitative data only for a fraction of the drug, which depends on the assay setup. These data may not reflect the true in vivo situation and may be difficult to correlate with PD or safety. Despite these limitations, assays quantifying target-binding competent drug reflect most of the PK assays actually used and described in filing documents and literature and should be appropriate assay types for many drugs also in future develop- 
ment studies; we are, therefore, of the opinion that defining a term for these type of assays would be beneficial, too. Another example where the targetbinding competent fraction does not equal the absolute amount of total drug is, when a certain amount of the drug is misfolded or degraded that abrogates binding to its target [9] or other degradation processes have occurred that abolish target-binding and, consequently, drug activity.

\section{"To determine the concentration of the drug fraction that is potent in its in vivo situation ... a binding protein or antidrug antibody in the binding region and molecules that are not functionally inactivated by degradation, we would propose to use the term 'active drug' to describe the respective analyte.}

Hybrid LC-MS/MS assays that use the target as affinity extraction tool can also be described to analyze 'target-binding competent drug' with the same principle considerations as described above.

\section{The definition of the term 'active drug'}

To determine the concentration of the drug fraction that is potent in its in vivo situation, namely drug molecules that are not complexed by soluble target, a binding protein or ADA in the binding region and molecules that are not functionally inactivated by degradation, we would propose to use the term 'active drug' to describe the respective analyte. Active drug assays are set up to quantify 'active drug' as close to the true in vivo situation as possible and are therefore most suited to establish correlation to functional readouts in vivo. This requires that the used assay formats reflect the mode of action of the drug. If the mode of action is described by target-binding, for example, for blocking or targeting monoclonal antibodies, an LBA can be used as 'active drug' quantification assay if the target is used for capture or detection of the drug. In addition, appropriate measures need to be undertaken to ensure that the equilibrium in vivo (e.g., between soluble target and drug) is not influenced during the assay procedure. This can be achieved by an appropriate assay conduct including a 'free-analyte QC concept,' which we proposed earlier [15]. Briefly, the free analyte $\mathrm{QC}$ concept requires that $\mathrm{QC}$ samples contain a defined concentration of both the soluble target as well as the drug in equilibrium prepared in a target-free matrix to verify the accuracy and precision of an assay; the calculation of the equilibrium and the resulting free drug concentration requires a correct value for $\mathrm{KD}$ in solution. Calibration is done by preparing a drug standard curve in a ligand-free matrix.

A previous theoretical assessment should, however, be done whether such an assay procedure is necessary. As described above, in situations where the level of soluble target is low compared with the anticipated drug dose and expected trough levels of the drug, a

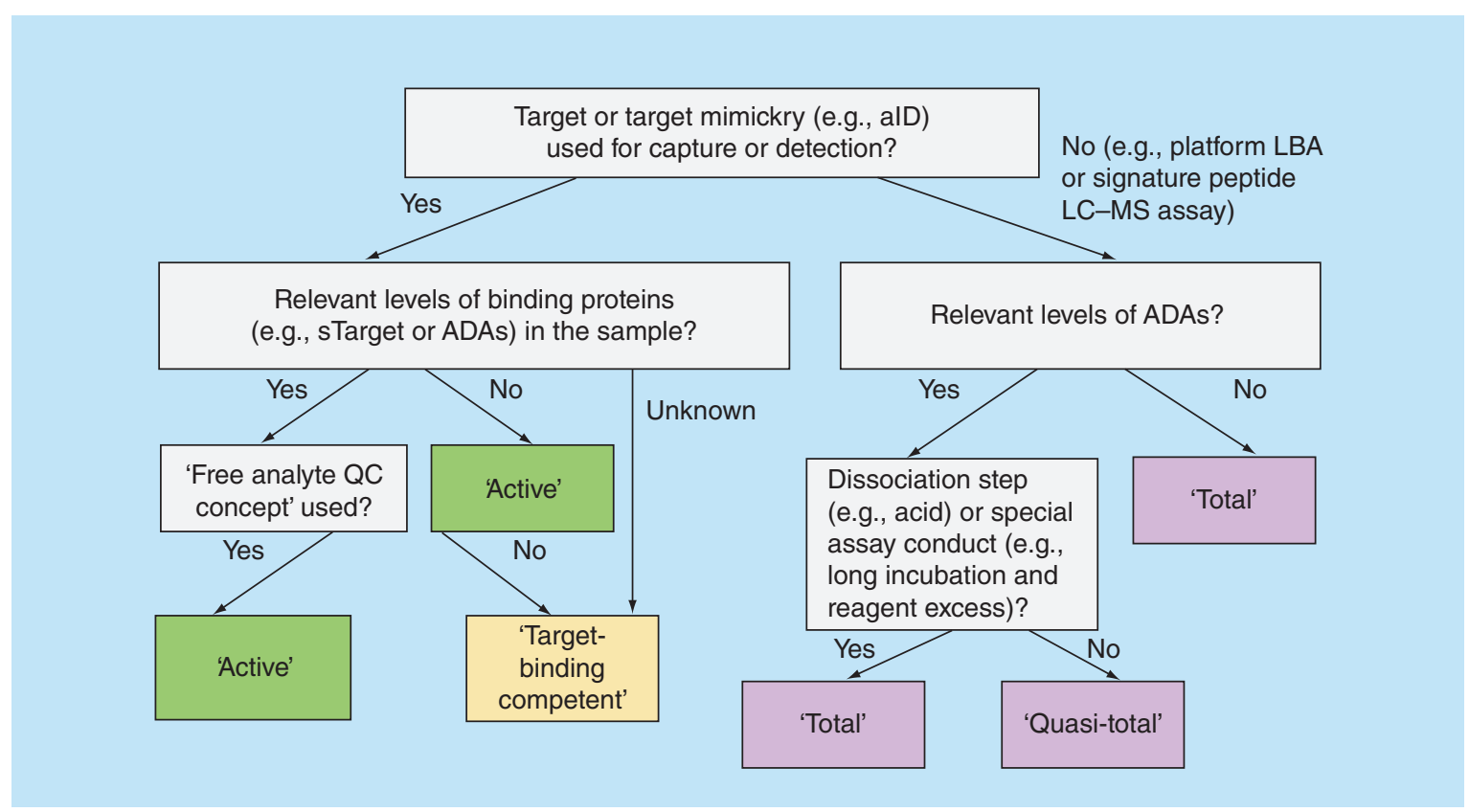

Figure 2. Decision tree to find the correct term for the analyte of an existing drug quantification assay. Refer to the text for detailed explanations.

ADA: Antidrug antibodies; aID: Anti-idiotypic antibody; LBA: Ligand-binding assays. 
less complex target-binding competent drug assay can be used and in approximation, the analyte can be described as 'active' drug [12]; however, in such cases the occurrence of ADAs needs to be monitored in parallel and ADA-positive subjects should be separated from negative subjects. Drug quantified in ADA-negative samples can be described as 'active drug,' whereas ADA-positive samples should then consequently be described as 'target-binding competent.'

Complexity further increases if activity of the drug requires bivalent binding (e.g., $\mathrm{mAbs}$ that execute mode of action mainly through avidity), an assay format needs to be used that allows for that assessment; this can, for example, be achieved by using a bridging ELISA that uses the soluble target for both, capture and detection which covers both functionalities.

In case a drug has multiple functions and each functionality is independent from each other to perform its mode of action, two separate assays would be required to proof activity of both functions. An example would be a bivalent bifunctional monoclonal antibody, such as the bispecific Crossmab antibody targeting both Ang2 and VEGF [16]. By contrast, in case a drug has multiple functionalities that are interdependent on each other, a single active drug assay is required, taking into consideration both functions. An example for such an analyte would be a targeting antibody or antibody-fragment toxin fusion protein for which mode of action is only achieved if both modalities are functional. Here, one could use a LBA assay capturing with the target and detecting with an antibody against the intact toxin domain to proof both functions in parallel [17]. As a general note, target-mimicries such as anti-idiotypic antibodies can be used equivalently, if the used anti-idiotypic antibodies have been characterized for their ability to mimic the target (e.g., by target competition assays and affinity determining assays or even functionalitybased assays) [18]. There are reports describing antiidiotypic antibodies that bind to the drug despite the drug having lost its ability to bind to the target. Therefore, a thorough characterization of the binding interaction is required [19].

\section{Conclusion \& outlook}

As initial guidance to find an accurate nomenclature for the analyte that is quantified in a defined assay setup, we have developed a decision tree (Figure 2); it is, however, obvious that it is advantageous to first define the form of the analyte that needs to be quantified and then design the assay accordingly.

The nomenclature that is proposed here targets at the discrimination of various forms of a drug analyte based on functionality. This enables a better understanding and interpretation of dose-response curves for efficacy or safety readouts. In that respect, we believe that special considerations should be taken when the term 'free drug' is used as this term can be misleading in certain cases as it does not describe the functionality of the drug. A drug molecule can, for example, be free, but degraded, rendering the drug inactive [9]. Also, a drug can be bound by a binding protein or an ADA and still exerts its function or a part of its function (e.g., nonbinding blocking ADAs or, for bispecific antibodies, ADAs blocking only one of the valencies).

Furthermore, but independent of the terms 'total,' 'target-binding competent' or 'active,' other, additional terms describing the integrity of the molecule may be helpful to describe the analyte quantified in a respective assay, especially for other complex biologics, such as ADCs, fusion proteins etc. These terms need to be carefully chosen considering the complex structure of proteins and usually require additional definition priori.

We are aware of the fact that the proposed categories cannot be regarded as absolute classifications due to the enormous microheterogeneity of biologics and their complexity in vivo. It is, however, our opinion that a discrimination from a functional perspective between total drug, target-binding competent and active drug will fit for the majority of bioanalytical assays and will help in providing clarity on the exact nature of the analyte, thus enabling better interpretation of bioanalytical data and improved communication of experts in the field of large-molecule bioanalysis. This publication is, therefore, meant to initiate a discussion on a descriptive nomenclature of bioanalytical analytes and, ultimately, leads to a clarification and consolidation within the bioanalytical community.

\section{Acknowledgements}

The authors thank the members of the Department of Large Molecule Bioanalytical R\&D for their valuable input and comments.

\section{Financial \& competing interests disclosure}

All authors are employees of Roche Diagnostics $\mathrm{GmbH}$. Some authors are owners of Roche bonus shares and profit certificates. The authors have no other relevant affiliations or financial involvement with any organization or entity with a financial interest in or financial conflict with the subject matter or materials discussed in the manuscript apart from those disclosed.

No writing assistance was utilized in the production of this manuscript. 


\section{References}

1 Dudal S, Staack RF, Stoellner D et al. How the bioanalyst plays a key role in interdisciplinary project teams in the development of biotherapeutics: a reflection of the European Bioanalysis Forum. Bioanalysis 6(10), 1339-1348 (2014).

2 Tatarewicz SM, Mytych DT, Manning MS, Swanson SJ, Moxness MS, Chirmule N. Strategic characterization of anti-drug antibody responses for the assessment of clinical relevance and impact. Bioanalysis 6(11), 1509-1523 (2014).

3 Schaefer M, Challand S, Schick E et al. An ex vivo potency assay to assess active drug levels of a GLP-1 agonistic peptide during preclinical safety studies. Bioanalysis doi:10.4155/ bio.15.189 (2015) (Epub ahead of print).

4 Hu J, Gupta S, Swanson SJ, Zhuang Y. A bioactive drug quantitation based approach for the detection of anti-drug neutralizing antibodies in human serum. J. Immunol. Methods 345(1-2), 70-79 (2009).

5 Lee JW, Kelley M, King LE et al. Bioanalytical approaches to quantify 'total' and 'free' therapeutic antibodies and their targets: technical challenges and PK/PD applications over the course of drug development. AAPS J. 13(1), 99-110 (2011).

6 Staack R, Jordan G, Viert M et al. Quantification of a bifunctional drug in the presence of an immune response: a ligand-binding assay specific for 'active' drug. Bioanalysis doi:10.4155/bio.15.213 (2015) (Epub ahead of print).

7 Kloks C, Berger C, Cortez P et al. A fit-for-purpose strategy for the risk-based immunogenicity testing of biotherapeutics: a European industry perspective. J. Immunol. Methods 417, 1-9 (2015).

8 Ponce R, Abad L, Amaravadi L et al. Immunogenicity of biologically-derived therapeutics: assessment and interpretation of nonclinical safety studies. Regul. Toxicol. Pharmacol. 54(2), 164-182 (2009).

9 Emrich T, Papadimitriou A. Characterization of asparagine deamidation in the CDR of a monoclonal antibody: monitoring of biotransformation in vitro and in vivo using high resolution ligand binding assays, selectively discriminating between active, inactive and total drug. Presented at: 9th Workshop on Recent Issues in Bioanalysis (9th WRIB). Miami, FL, USA, 13-17 April 2015.
10 Kuang B, King L, Wang HF. Therapeutic monoclonal antibody concentration monitoring: free or total? Bioanalysis 2(6), 1125-1140 (2010).

11 Kelley M, Ahene AB, Gorovits B et al. Theoretical considerations and practical approaches to address the effect of anti-drug antibody (ADA) on quantification of biotherapeutics in circulation. AAPS. J. 15(3), 646-658 (2013).

12 Staack RF, Jordan G, Heinrich J. Mathematical simulations for bioanalytical assay development: the (un-)necessity and (im-)possibility of free drug quantification. Bioanalysis 4(4), 381-395 (2012).

13 Stubenrauch K, Wessels U, Lenz H. Evaluation of an immunoassay for human-specific quantitation of therapeutic antibodies in serum samples from non-human primates. J. Pharm. Biomed. Anal. 49(4), 1003-1008 (2009).

14 Stubenrauch K, Wessels U, Essig U, Kowalewsky F, Vogel $\mathrm{R}$, Heinrich J. Characterization of murine anti-human Fab antibodies for use in an immunoassay for generic quantification of human Fab fragments in non-human serum samples including cynomolgus monkey samples. J. Pharm. Biomed. Anal. 72, 208-215 (2013).

15 Staack RF, Jordan G, Dahl U, Heinrich J. Free analyte QC concept: a novel approach to prove correct quantification of free therapeutic protein drug/biomarker concentrations. Bioanalysis 6(4), 485-496 (2014).

16 Schaefer W, Regula JT, Bahner M et al. Immunoglobulin domain crossover as a generic approach for the production of bispecific IgG antibodies. Proc. Natl Acad. Sci. USA 108(27), 11187-11192 (2011).

17 Alewine C, Xiang L, Yamori T, Niederfellner G, Bosslet K, Pastan I. Efficacy of RG7787, a next-generation mesothelintargeted immunotoxin, against triple-negative breast and gastric cancers. Mol. Cancer Ther. 13(11), 2653-2661 (2014).

18 Chin SE, Ferraro F, Groves M, Liang M, Vaughan TJ, Dobson CL. Isolation of high-affinity, neutralizing anti-idiotype antibodies by phage and ribosome display for application in immunogenicity and pharmacokinetic analyses. J. Immunol. Methods 416, 49-58 (2015)

19 Salimi-Moosavi H, Winters A, Abbott C et al. A multifactorial screening strategy to identify anti-idiotypic reagents for bioanalytical support of antibody therapeutics. Anal. Biochem. 470, 52-60 (2015). 